\title{
Gene-gene Interaction Between EPAS1 and EGLN1 in Patients with High-Altitude Pulmonary Edema
}

\author{
Toshimichi Horiuchi ${ }^{1)}$, Masao OTA ${ }^{2)}$, Yunden Droma ${ }^{1)}$ \\ Nobumitsu KobaYASHi ${ }^{1)}$ and Masayuki HanaokA ${ }^{1) *}$ \\ 1) The First Department of Medicine, Shinshu University School of Medicine \\ 2) Department of Legal Medicine, Shinshu University School of Medicine
}

\begin{abstract}
Objective : Endothelial PER-ARNT-SIM (PAS) domain protein 1 (EPAS1, also known as HIF2 $\alpha$ ) and egl nine homolog 1 (EFLN1), also known as prolyl hydroxylase domain protein 2 (PHD2), have key functions in the upstream of the hypoxia-inducible factor (HIF) pathway. In order to dissect the genetic biology of susceptibility to high-altitude pulmonary edema (HAPE) from the viewpoint of the HIF pathway, we identified the allelic discriminations of three significant tag single-nucleotide polymorphisms (SNPs) in EPAS1 and three tag SNPS in EGLN1 in HAPE-susceptible (HAPE-s) Japanese subjects.
\end{abstract}

Methods: Alleles were determined for the six SNPs (rs13419896, rs4953354, and rs4953388 in EPAS1; rs1435166, rs7542797, and rs2153364 in EGLN1) by the TaqMan ${ }^{\circledR}$ SNP Genotyping Assay in a group of 59 HAPE-s subjects and a control group of 67 HAPE resistant (HAPE-r) subjects. In addition to the case-control analysis, multi-dimensional reduction (MDR) methodology was applied to a gene-gene interaction analysis to evaluate the association of HAPE-s with gene-gene interactions.

Results : The EGLN1 rs2153364 (A/G) x EPAS1 rs13419896 (G/A) interaction was significantly associated with HAPE-s in the pairwise model $(\mathrm{P}=0.0049)$ based on the balanced accuracy of $63.23 \%$ in MDR. However, no significance was detected for the association with $\mathrm{HAPE}_{-\mathrm{s}}$ in the single gene model.

Conclusion: The EPAS1-EGLN1 interaction appears to be associated with HAPE-s in the Japanese population despite the individual genes not being associated with HAPE-s. Shinshu Med J 63 : 157-165, 2015

(Received for publication January 29, 2015 ; accepted in revised form February 17, 2015)

Key words : hypoxia-inducible factor, gene-gene interaction, high-altitude pulmonary edema

\section{Introduction}

Mountains cover more than one-fifth of the Earth's surface and are popular tourist destinations. Large numbers of people travel to high altitudes for various reasons, including trekking, pilgrimage, and tourism. In Japan, approximately 38-65 million people climb mountains ( $>2,000 \mathrm{~m}$ above sea level) for recreation each year according to the Japan

\footnotetext{
* Corresponding author: Masayuki Hanaoka

The First Department of Medicine, Shinshu University School of Medicine, 3-1-1 Asahi, Matsumoto, Nagano 390-8621, Japan

E-mail : masayuki@shinshu-u.ac.jp
}

Science Research Society (http://soukaken.com/,in Japanese). Exposure to the oxygen-depleted environment triggers the onset of a range of physiological and biochemical reactions that enhance the efficacy of the body's respiratory ${ }^{1)}$, cardiovascular, and oxygen utilization systems ${ }^{2)}$ through the process of acclimatization. Failure to acclimatize leads to high-altitude pulmonary edema (HAPE), a form of accelerated pulmonary edema of non-cardiogenic origin that occurs in lowlanders who rapidly ascend above $2,500 \mathrm{~m}$; it has a considerably high mortality if hypoxic exposure persists ${ }^{1-3)}$. The inception and progression of HAPE are governed by both genetic and environmental factors ${ }^{4) 5}$. 
The hypoxia-inducible factor (HIF) pathway triggers downstream cascades of multiple molecular events involving the pathophysiology of hypoxia ${ }^{6}$. More than a dozen candidate genes in the HIF pathway have been shown to contribute to the genetic adaptation to hypoxia in high-altitude populations across multiple studies ${ }^{7)-10)}$, including endothelial PER-ARNT-SIM (PAS) domain protein 1 (EPAS1), also known as HIF2 $\alpha$, and egl nine homolog 1 (EGLN1), also known as prolyl hydroxylase domain protein 2 (PHD2). These two key genes function in the upstream of the HIF pathway in human high-altitude adaptation ${ }^{11-15)}$. Specifically, the single-nucleotide polymorphisms (SNPs) of rs13419896 (G/A), rs4953354 (A/G), and rs4953388 (G/A) in EPAS1 were demonstrated to be significantly associated with hypoxia tolerance in indigenous high-altitude Tibetan $^{7)}$ and Sherpa ${ }^{16)}$ populations. Moreover, the SNPs of rs1435166 (A/ $\mathrm{G}), \operatorname{rs} 7542797(\mathrm{~A} / \mathrm{C}), \operatorname{rs} 2153364(\mathrm{~A} / \mathrm{G})$ in EGLN1 were documented to be significantly associated with human high-altitude adaptation in the Indo-Aryan population ${ }^{14)}$. Obviously, the genetic contributions of HIF to hypoxia adaptation in indigenous high-altitude populations may not be applicable to the elucidation of genetic biology in HAPE, an acute disease caused by sudden oxygen deprivation, despite highaltitude hypoxia being a common initial element in both disorders. Indigenous high-altitude populations, such as Tibetans and Sherpas, have successfully lived and reproduced at high altitudes for hundreds of generations with hypoxia as a constant evolutionary pressure, bringing about natural selection toward phenotypes (e.g., relatively low hemoglobin) that tend to offer beneficial adaptations to sustained hypoxia ${ }^{17) 18}$. In contrast, HAPE occurs in lowlanders rapidly exposed to a high altitude, resulting in a failure to acclimatize to high-altitude hypoxia, which is characterized by hypoxia-induced acute pulmonary vasoconstriction ${ }^{2)-4)}$.

In order to dissect the genetic biology of HAPE susceptibility from the viewpoint of the HIF pathway, we identified the allelic discriminations of three significant tag SNPs in EPAS1 (rs13419896, rs4953354, and rs4953388) and three tag SNPs in EGLN1 (rs1435166, rs7542797, and rs2153364) in HAPE-susceptible (HAPE-s) Japanese subjects and compared the results to HAPE-resistant (HAPE-r) Japanese subjects in a case- control study and gene- $^{-}$ gene interaction analysis.

\section{Methods}

\section{A Ethics statement}

The current study was approved by the Ethics Committee of Shinshu University School of Medicine (Matsumoto, Japan). The protocol was performed in accordance with the principles outlined in the Declaration on Helsinki of the World Medical Association $^{19)}$ and was approved by the Ethics Committee of Shinshu University School of Medicine (Permission numbers: 106). Written informed consent was obtained from each subject after providing them with a full explanation of the study.

\section{B Subjects}

The case-control study included a case group with 59 HAPE-s subjects (52 males, 7 females, average age 34.2 years) and a control group with 67 HAPE-r subjects (58 males, 9 females, average age 37.0 years). All subjects were unrelated natives born in Japan and resided at low altitudes. The HAPE-s subjects were patients admitted to Shinshu University Hospital because of HAPE that occurred while climbing in the Japan Alps at heights over 2,500 m between 1971 and 2013. The diagnosis of HAPE was based on diagnostic criteria at the onset of the disorder ${ }^{20)}$. All patients with HAPE recovered promptly within one week of hospitalization. Related clinical examinations and cardiovascular tests were conducted to exclude any pre-existing cardiopulmonary problems. The HAPE-r subjects were elite mountaineers from the Mountaineering Association of Nagano Prefecture (Nagano, Japan) and the Alpine Club of Shinshu University (Matsumoto, Japan) and often climbed mountains higher than 3,000 m. We defined these subjects as HAPE-r due to their resistance to HAPE during exposure to high-altitude environments. During recruitment, no HAPE-r subjects reported a history of medical problems related 
to altitude or cardiopulmonary disorders in a questionnaire containing the components of the Lake Louise Score $^{21)}$. Venous blood samples were collected and frozen at $\leq-70{ }^{\circ} \mathrm{C}$ for research purposes.

\section{Selection of EPAS1 Tag SNPs}

The tag SNPs act as direct proxies for all other untyped SNPs because they are highly correlated with one another by means of pairwise tagging ${ }^{22)}$. Setting the threshold of correction coefficient $\left(\mathrm{r}^{2}\right)$ at 1.0 resulted in a perfect correlation between the tag SNP and untyped SNPs, capturing the untyped SNPs perfectly and efficiently.

The human EPAS1 gene contains 16 exons, with spans of at least $120 \mathrm{~kb}$, and maps to chromosome $2 \mathrm{p} 21-\mathrm{p} 16$. The introns within the genomic region encode the N-terminal basic helix-loop-helix (bHLH)/PAS proteins, which are critical regulators of gene expression networks of transcriptional responses to low oxygen tension mediated by the HIF signaling pathway ${ }^{23)}$. Three SNPs [rs13419896 (G, ancestral allele/A, derived allele) according to the NCBI dbSNP database, rs4953354 (A/G), and rs4953388 (G/A)] in EPAS1 were selected as tag SNPs because they can capture $\left(\mathrm{r}^{2}=1.0\right)$ numerous untyped SNPs in EPAS1 from the genotype data in the International HapMap Consortium 2005 (http:// hapmap.ncbi.nlm.nih.gov/) with the Japanese population from Tokyo, Japan (JPT) (Table 1). In addition, these SNPs are significantly associated with hypoxia tolerance in indigenous high-altitude Tibetan and Sherpa populations ${ }^{7) 16}$. All three SNPS are located in introns.

\section{Selection of EGLN1 Tag SNPs}

The EGLN1 gene contains four coding exons and maps to chromosome 1q42-q43. Three SNPs [rs1435166 (A/G), rs7542797 (A/C), and rs2153364 $(\mathrm{A} / \mathrm{G})]$ were selected as tag SNPs due to an evaluation of their tagging efficiency $\left(\mathrm{r}^{2}=1.0\right)$ for available SNPS in EGLN1 from the genotype data in the International HapMap Consortium 2005 (http://hapmap.ncbi.nlm.nih.gov/) with the JPT population (Table 2). In particular, EGLN1 rs1435166 (Table 2) had highly pairwise correlations with seven SNPs reported to be significantly associated with $\mathrm{HAPE}-$ $\mathrm{S}$ in the Indian population ${ }^{14)}$. All three SNPs are located in introns.

\section{E Genotyping}

Genomic DNA samples from HAPE-s and HAPE-r subjects were extracted from venous blood leukocytes as described previously ${ }^{5) 16}$. The SNP Genotyping Assay Mix containing the forward and reverse primers and $\mathrm{FAM}^{\mathrm{TM}}$ and $\mathrm{VIC}^{\mathrm{TM}}$ dye-minor groove binder-labeled probes for the six SNPs were purchased from Applied Biosystems Inc. (Tokyo, Japan). Allelic discrimination was performed using a pre-designed $5^{\prime}$ nuclease assay (TaqMan ${ }^{\circledR}$ SNP Genotyping Assay) and the Applied Biosystems 7500 Fast Real-time PCR System according to the manufacturer's instructions (Applied Biosystems Inc., Foster City, CA, USA). Following thermal cycling, genotype data were acquired automatically and analyzed using sequence detection software (SDS

Table 1 Tagging efficiency of single nucleotide polymorphisms (SNPs) rs13419896 (G/A), rs4953354 (A/G), and rs4953388 (G/A) in EPAS1 in the Tokyo, Japan (JPT) population*. HapMap-JPT

\begin{tabular}{ll}
\hline Test SNP & Alleles captured** \\
\hline \hline rs13419896 & rs4952819, rs4953342, rs4952820, and rs4953348 \\
rs4953354 & rs10187368, rs4953355, rs1868085, rs1868086, rs1868087, rs1374748, and rs13409493 \\
& rs1447563, rs4953372, rs11125075, rs1530624, rs4396767, rs12986899, rs13003074, rs2121700, rs718849, \\
& rs13020043, rs13002880, rs2121698, rs4953387, rs4953390, rs13030579, rs12986653, rs13001507, rs13007688, \\
& rs13011721, rs6544898, rs13005507, rs13024277, rs13024546, rs13025211, rs17817004, rs4953396, rs2084679, \\
& rs13010097, rs2346414, rs2346415, rs880671, and rs4952830 \\
\hline
\end{tabular}

*Checked by tagger software based on the genotype data in the International HapMap Consortium 2005 (http:// hapmap.ncbi.nlm.nih.gov/) with Japanese from the JPT.

**The threshold of pairwise correction $\left(\mathrm{r}^{2}\right)$ was set at 1.0, at which all alleles are to be captured. 
Table 2 Tagging efficiency of single nucleotide polymorphisms (SNPs) rs1435166 (A/G), rs7542797 (A/C), and rs2153364 (A/G) in EGLN1 in the Tokyo, Japan (JPT) population*.

HapMap-JPT

\begin{tabular}{ll}
\hline Test SNP & Alleles captured** \\
\hline \hline & rs479200, rs2486729, rs2437148, rs2808614, rs2244994, rs2486732, rs2024878, rs10489611, rs2437147, rs973254, \\
& rs2472261, rs2790889, rs2486736, rs2749699, rs2808616, rs2808584, rs2808586, rs2790890, rs2486731, \\
rs1435166 & rs2486742, rs2790887, rs2749710, rs480902, rs2066140, rs10489610, rs973252, rs2486727, rs2244986, rs2790879, \\
& rs2474623, rs973253, rs2808611, rs2739513, rs2486737, rs2572263, rs1538664, rs2808580, rs2486741, rs2739511, \\
& rs2491405, rs2491404, rs2790891, rs2790882, and rs2486745 \\
rs7542797 & rs7542797 \\
rs2153364 & rs2153364 \\
\hline
\end{tabular}

${ }^{*}$ Checked by tagger software based on the genotype data in the International HapMap Consortium 2005 (http:// hapmap.ncbi.nlm.nih.gov/) with Japanese from the JPT.

**The threshold of pairwise correction $\left(\mathrm{r}^{2}\right)$ was set at 1.0, at which all alleles are to be captured. The SNPs in bold were reported to be significantly associated with $\mathrm{HAPE}^{-\mathrm{S}}$ in the Indian population (14).

\section{v1.3.1, Applied Biosystems Inc.).}

\section{F Statistical analysis}

The chi-square test was used for between-group comparisons, but Fisher's exact probability test was used for comparisons when the number of subjects was less than five. The exact test of Hardy-Weinberg equilibrium (HWE) was performed by the Markov chain method, which is reported to have the advantage of obtaining complete enumeration when the number of alleles and sample size are small ${ }^{24}$. Therefore, the possibility of type II error due to our relatively small sample sizes was minimized. Twotailed $\mathrm{P}-$ values $<0.05$ indicated significance. The current genetic analysis was trustworthy despite the relatively small sample sizes in both groups because the studied cohorts were ethnically homogenous Japanese.

Gene-gene interactions were evaluated by multidimensional reduction (MDR, version 3.0.2) with the variable alleles of the SNPs in the two genes. Random combinations of pairwise SNPs were analyzed by MDR (http://www.multifactordimensionalityreduction.org/) to detect and characterize the pairwise interactions of these SNPs across the two genes. The relative risk (RR) and $95 \%$ confidence interval (95\% CI) were calculated accordingly.

\section{Results}

\section{A Single gene model}

The genotype distributions of the six SNPs conformed to HWE $(\mathrm{P}>0.05)$ in both the case and control groups. No significant differences were detected between the case and control groups in terms of genotype distributions or allelic frequencies for the six SNPs (Table 3), suggesting that these SNPs were not associated with HAPE susceptibility in the single gene model.

\section{B Gene-gene interaction analysis}

Because three SNPs (EPAS1 rs4953354 and rs4953388 and EGLN1 rs7542797) were not derived homozygous genotypes in the HAPE-s and HAPEr groups (Table 3), the EGLN1 rs2153364 (A/G) x EPAS1 rs13419896 (G/A) model was the best model of random pairwise combinations for the six SNPs across the two genes, based on a balanced accuracy of $63.23 \%$ and a cross-validation consistency of $6 /$ 10 in the performance of MDR in the whole dataset (Fig. 1). This best pairwise interaction model (Fig. 1) showed a significant association with $\mathrm{HAPE}_{-\mathrm{S}}$ $[\mathrm{P}=0.0049$; $95 \%$ confidence interval (CI) 3.16 (1.407.14)]. Regarding HAPE susceptibility, the interaction between EGLN1 rs2153364 AA (ancestral genotype) and EPAS1 rs13419896 GA (heterozygous genotype) was categorized as the most high-risk combination (9 subjects of HAPE-s vs. 3 subjects of 
EPAS1 and EGLN1 interaction in high-altitude pulmonary edema

Table 3 Genotype distributions and allele frequencies of rs13419896, rs4953354, and rs4953388 in EPAS1 and rs1435166, rs7542797, and rs2153364 in EGLN1 in HAPE-s $(\mathrm{n}=59)$ and HAPE-r subjects $(\mathrm{n}=67)$

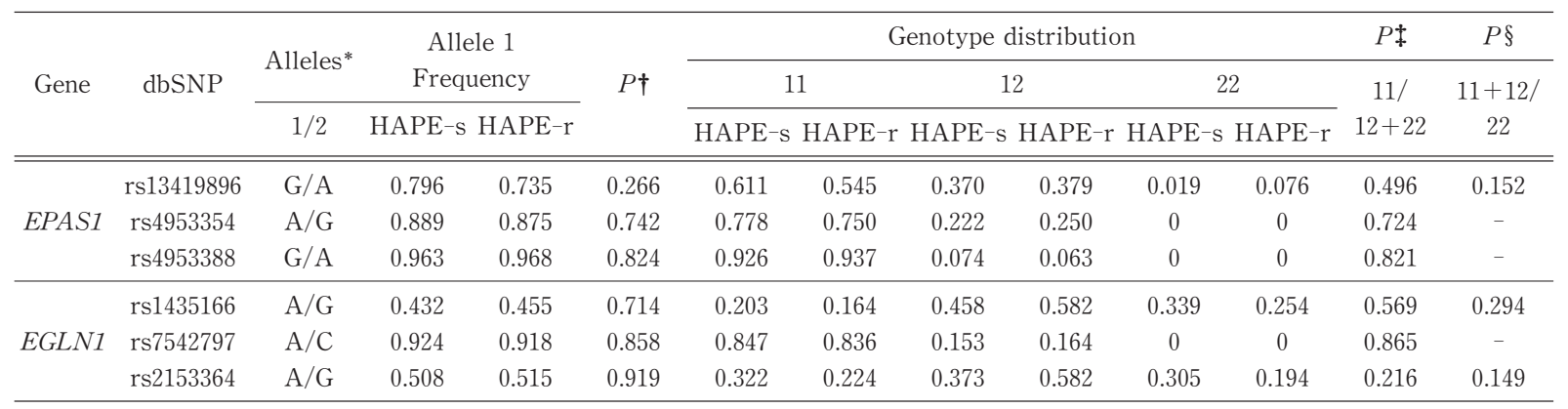

$\mathrm{n}=$ total number of subjects $\mathrm{SNP}=$ single nucleotide polymorphism ; HAPE-s $=$ subjects susceptible to high-altitude pulmonary edema ;

$\mathrm{HAPE}-\mathrm{r}=$ subjects resistant to high-altitude pulmonary edema.

${ }^{*} 1 / 2$ indicates ancestral allele/derived allele according to the NCBI dbSNP database.

$\dagger$ Chi-square test $(2 \times 2$ contingency table).

$\$ 2 \times 2$ contingency table assuming dominant mode $(11 / 12+22)$ of inheritance in HAPE- .

$\S 2 \times 2$ contingency table assuming recessive mode $(11+12 / 22)$ of inheritance in HAPE-s.

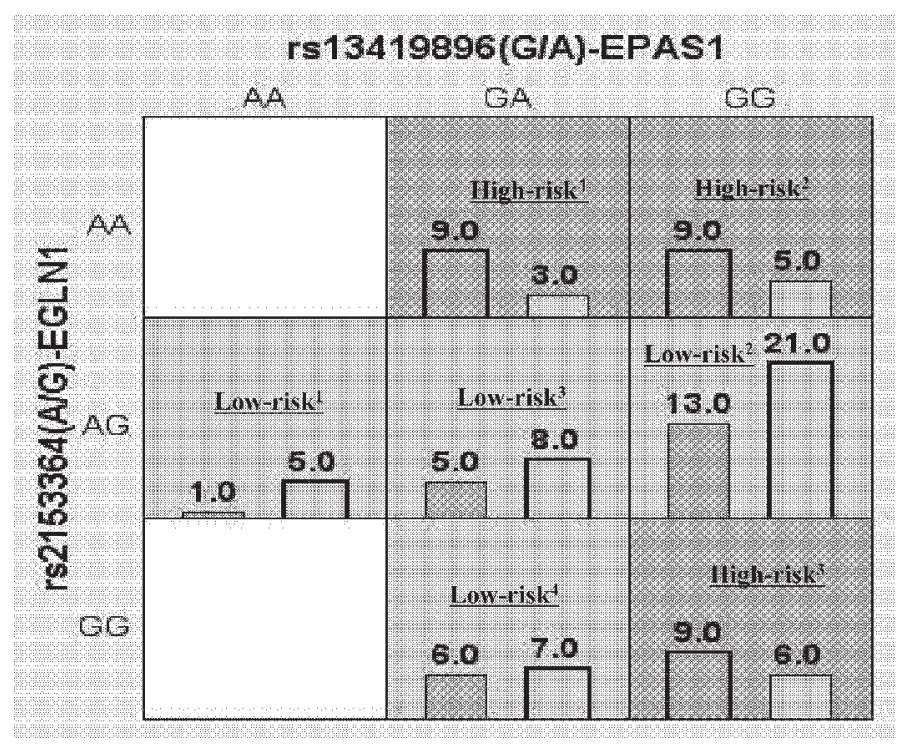

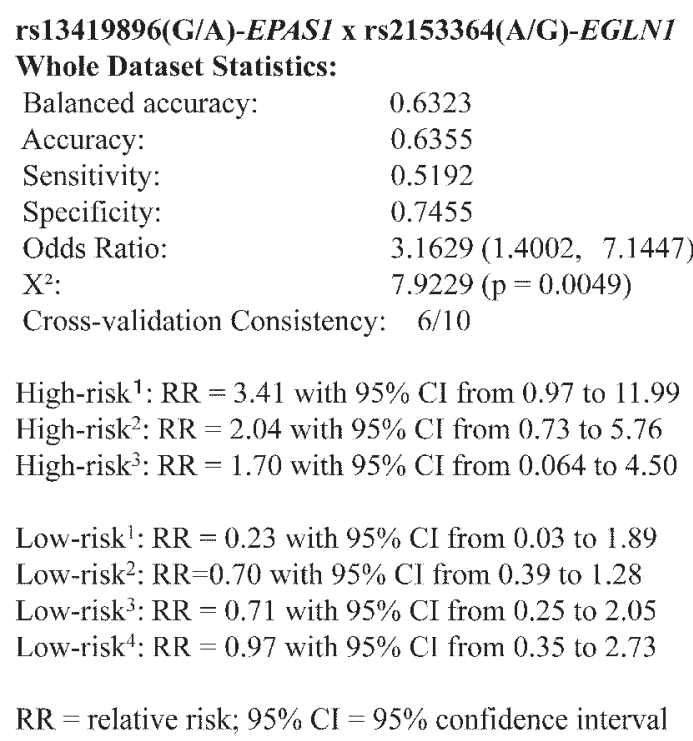

Fig. 1 The best model as determined by multi-dimensional reduction (MDR) for EPAS1 rs13419896 (G/ A) x EGLN1 rs2153364 (A/G). The numbers within each square represent the numbers of cases (left) and controls (right). For each square, dark-grey shading indicates a high risk of highaltitude pulmonary edema (HAPE), whereas light-grey shading represents a low risk of HAPE and white shading indicates no combination. The interaction between EGLN1 rs2153364 AA and EPAS1 rs13419896 GA was categorized as the most high-risk combination for HAPE susceptibility [relative risk $(\mathrm{RR})=3.41$ with $95 \%$ confidence interval $(95 \% \mathrm{CI})$ from 0.97 to 11.99 ], whereas the interaction between EGLN1 rs2153364 AG and EPAS1 rs13419896 AA was the most low-risk combination for HAPE susceptibility in the Japanese population $(\mathrm{RR}=0.23$ with $95 \%$ CI from 0 . 03 to 1.89). The other high-risk combinations were EGLN1 rs2153364 AA x EPAS1 rs13419896 GG $(\mathrm{RR}=2.04$ with $95 \% \mathrm{CI}$ from 0.73 to 5.76) and EGLN1 rs2153364 GG x EPAS1 rs13419896 GG $(\mathrm{RR}=1.70$ with $95 \% \mathrm{CI}$ from 0.064 to 4.50$)$, whereas the other low-risk combinations were EGLN1 rs2153364 AG x EPAS1 rs13419896 GG (RR=0.70 with $95 \%$ IC from 0.39 to 1.28), EGLN1 rs2153364 AG x EPAS1 rs13419896 GA (RR=0.71 with $95 \%$ IC from 0.25 to 2.05) and EGLN1 rs2153364 GG x EPAS1 rs13419896 GA (RR=0.97 with $95 \%$ IC from 0.35 to 2.73 ). 
HAPE-r, RR=3.41 with $95 \%$ CI from 0.97 to 11.99) among the detected three high-risk combinations, whereas the interaction between EGLN1 rs2153364 AG (heterozygous genotype) and EPAS1 rs13419896 AA (ancestral genotype) was the most low-risk combination (1 subjects of HAPE-s vs. 5 subjects of HAPE-r, RR $=0.23$ with $95 \%$ CI from 0.03 to 1.89) among the four detected low-risk combinations of genotypes in the present Japanese population (Fig. 1).

\section{Discussion}

The main result of the present study was the detection of a potential association of the interaction between EPAS1 and EGLN1 with HAPE susceptibility in the Japanese population. The subjects carrying the AA ancestral genotype of EGLN1 rs2153364 (A/G) and the GA heterozygous genotype of EPAS1 rs13419896 (G/A) were at high risk for HAPE. The present study provides some suggestive information about the EPAS1 and EGLN1 genes in association with HAPE susceptibility in regards to the HIF signaling pathway. However, these two genes did not show an association with $\mathrm{HAPE}^{-\mathrm{s}}$ in the Japanese population in the single gene model.

EPAS1 encodes the hypoxia-inducible-factor-2 alpha (HIF2A) protein, which shares $48 \%$ sequence identity with HIF1A ${ }^{23)}$. Similar to HIF1A, EPAS1 is stimulated by hypoxic conditions and heterodimerizes with HIF2B for transcriptional activation of target genes ${ }^{6)}$. In contrast, EGLN1 encodes prolyl hydroxylase domain protein 2 (PHD2), which senses hypoxia and negatively affects the activity of HIF2A and plays a regulatory role in mammalian oxygen homeostasis ${ }^{25)}$. In normoxia, HIF2A subunits are marked for the ubiquitin-proteasome degradation pathway through hydroxylation of proline by PHD2. When exposed to hypoxia, the PHD2-dependent hydroxylation is inadequate and HIF2A marks for ubiquitin-proteasome degradation fade away, thereby enhancing the HIF signaling pathway and inducing the expression of a series of genes that activate glycolytic enzymes, hemeoxygenase, vascular endothelial growth factor, and erythropoietin, among others, to challenge hypoxia stress physiologically ${ }^{6) 23(25)}$.

Over the years, a body of evidence has accumulated for the genetic loci of EPAS1 and EGLN1 being associated with human adaptation to high-altitude hypoxia through generations ${ }^{7)-15)}$. The three SNPs [rs13419896 (G/A), rs4953354 (A/G), rs4953388 (G/ A)] in EPAS1 are significantly associated with hypoxia tolerance in indigenous high-altitude Tibetan $^{7)}$ and Sherpa ${ }^{16)}$ populations. In addition, the three SNPs $[\operatorname{rs} 1435166(\mathrm{~A} / \mathrm{G}), \operatorname{rs} 7542797$ (A/C), rs2153364 (A/G)] in EGLN1 highly correlate with rs479200, rs2486729, rs2486736, rs480902, rs2790879, rs973253, and rs1538664 in EGLN1 in the Japanese population; these SNPs have been significantly associated with HAPE-s subjects in the Indo-Aryan population ${ }^{14)}$. However, the present study did not show any associations between the six SNPs and HAPE susceptibility in the Japanese population in the single gene model. On the other hand, the genegene interaction analysis suggested a potential association of the EPAS1-EGLN1 interaction with HAPE susceptibility in the Japanese population. The adaptive physiological features of Tibetans, such as a low hypoxic pulmonary vasoconstrictor response and relatively low hemoglobin levels, were acquired through natural selection over generations, as the ancestors of Tibetans occupied the highaltitude region as early as 30,000 years $\mathrm{ago}^{17}$. In contrast, the pathophysiological features of HAPE characterized by acute hypoxia-induced pulmonary vasoconstriction and permeable pulmonary vascular endothelium occur acutely in lowlanders rapidly exposed to high altitudes and hypoxia ${ }^{1)-4)}$. Thus, the process of natural selection in the Tibetan adaptation to hypoxia is distinct from the pathogenesis of an acute challenge to hypoxic stress in lowlanders rapidly exposed to high altitudes. Regarding the significant associations of EGLN1 loci with susceptibility to HAPE in the Indo-Aryan population ${ }^{14}$, the probability of replicating such results is only occasional in the Japanese population because of the polygenic architecture of the human genome ${ }^{26)}$ and the characteristics of $\mathrm{SNPs}^{27)}$. Nevertheless, 
EGLN1 rs480902 did not show a significant association with acute mountain sickness in the Han Chinese population ${ }^{15)}$.

Epistasis, or interactions between genes, is fundamentally important to understanding both the structure and function of genetic pathways and the evolutionary dynamics of complex genetic systems ${ }^{28)}$. The methodology of gene-gene interaction analysis was eventually carried out to assess the multidimensional relations of the EPAS1 and EGLN1 genes of the HIF signaling pathway with HAPE susceptibility in the Japanese population in the present study, despite the fact that numerous candidate genes were individually identified as being significantly associated with HAPE-s populations worldwide ${ }^{29)-32}$. The current superficial results are not direct evidence of the functions of and communications between the genetic varieties for the physiological response to acute hypoxia in HAPE susceptibility. However, the present results showing that the ancestral homozygous AA genotype of EGLN1 rs2153364 (A/G) interacts with EPAS1 rs13419896 (G/A) as a high-risk genotype in HAPE-s subjects is in agreement with the previous report by Mishar et al.; they reported a 4.55 -fold up-regulation of EGLN1 expression in HAPE-s subjects compared to HAPE-r subjects ${ }^{14}$. Therefore, we proposed that the up-regulation of the negative regulator of EGLN1 causes down-regulation of proteasome degradation of HIF-2 in a hypoxic environment, leading to HIF-2 accumulation and inducing overexpressions of downstream genes that activate glycolytic enzymes, hemeoxygenase, vascular en- dothelial growth factor, and erythropoietin, among others, to participate in the pathogenesis of HAPE. Detailed investigations are expected to determine the functions of the gene-gene interactions in the HIF signaling pathway in the pathogenesis of HAPE.

The power of the present study was inadequate due to the limitations of the MDR method, which reduced high-dimensional genetic data into a single dimension so that gene-gene interactions could be detected in a sample size smaller than the original sample size. HAPE is actually a relatively rare disease and occurs only in lowlanders rapidly exposed to high altitudes, so the sample size is always smaller than other common diseases. Thus, the present gene-gene interaction analysis needs to be replicated in other populations with HAPE and proven by other technical analyses of gene-gene interactions, such as BOOST ${ }^{33)}$.

In conclusion, the present study demonstrated a potential association of the EPAS1 $\times E G L N 1$ interaction with HAPE susceptibility in the Japanese population, despite no associations detected in the single gene model. This finding is small, but encouraging, and needs to be validated through replication studies with large sample sizes and different ethnicities to establish the global efficacy of these genes in the HIF signaling pathway regarding hypoxic response in HAPE susceptibility.

The authors state that thay have no conflict of interest in the present study.

\section{References}

1) Smith CA, Dempsey JA, Hornbein TF : Control of breathing at high altitude. In : Hornbein TF, Schoene RB (eds), High altitude: an exploration of human adaptation, pp 525-568, Marcel Dekker, New York, 2001

2) Hohenhaus E, Paul A, McCullough RE, Kucherer H, Bartsch P : Ventilatory and pulmonary vascular response to hypoxia and susceptibility to high altitude pulmonary oedema. Eur Respir J 8: 1825-1833, 1995

3) Maggiorini M : High altitude-induced pulmonary oedema. Cardiovasc Res $72: 41-50,2006$

4) Hackett PH, Roach RC: High-altitude illness. N Engl J Med 345 : 107-114, 2001

5) Kobayashi N, Hanaoka M, Droma Y, Ito M, Katsuyama Y, Kubo K, Ota M : Polymorphisms of the tissue inhibitor of metalloproteinase 3 gene are associated with resistance to high-altitude pulmonary edema (HAPE) in a Japanese population: A case control study using polymorphic microsatellite markers. PLoS ONE, 8 : e71993. doi : 
10.1371/journal. pone. 0071993, 2013

6) Semenza GL: Regulation of oxygen homeostasis by hypoxia-inducible factor 1. Physiology (Bethesda) 24: 97-106, 2009

7) Beall CM, Cavalleri GL, Deng L, Elston RC, Gao Y, Knight J, Li C, Li JC, Liang Y, McCormack M, Montgomeryi HE, Pan H, Robbins PA, Shianna KV, Tam SC, Tsering N, Veeramah KR, Wang W, Wangdui P, Weale ME, Xu Y, Xu Z, Yang L, Zaman MJ, Zeng C, Zhango L, Zhang X, Zhanxi P, Zheng YT: Natural selection on EPAS1 $(H I F 2 \alpha)$ associated with low hemoglobin concentration in Tibetan highlanders. Proc Natl Acad Sci U S A 107 : 11459-11464, 2010

8) Simonson TS, Yang Y, Huff CD, Yun H, Qin G, Witherspoon DJ, Bai Z, Lorenzo FR, Xing J, Jorde LB, Prchal JT, Ge R: Genetic evidence for high-altitude adaptation in Tibet. Science 329: 72-75, 2010

9) Simonson TS, McClain DA, Jorde LB, Prchal JT : Genetic determinants of Tibetan high-altitude adaptation. Hum Genet $131: 527-533,2012$

10) Yi X, Liang Y, Huerta-Sanchez E, Jin X, Cuo ZX, Pool JE, Xu X, Jiang H, Vinckenbosch N, Korneliussen TS, Zheng H, Liu T, He W, Li K, Luo R, Nie X, Wu H, Zhao M, Cao H, Zou J, Shan Y, Li S, Yang Q, Asan, Ni P, Tian G, Xu J, Liu X, Jiang T, Wu R, Zhou G, Tang M, Qin J, Wang T, Feng S, Li G, Huasang, Luosang J, Wang W, Chen F, Wang Y, Zheng X, Li Z, Bianba Z, Yang G, Wang X, Tang S, Gao G, Chen Y, Luo Z, Gusang L, Cao Z, Zhang Q, Ouyang W, Ren X, Liang H, Zheng H, Huang Y, Li J, Bolund L, Kristiansen K, Li Y, Zhang Y, Zhang X, Li R, Li S, Yang H, Nielsen R, Wang J, Wang J : Sequencing of 50 human exomes reveals adaptation to high altitude. Science $329:$ 75-78, 2010

11) Bigham AW, Lee FS : Human high-altitude adaptation : forward genetics meets the HIF pathway. Genes Dev 28 : 2189-2204, 2014

12) Song D, Li LS, Arsenault PR, Tan Q, Bigham AW, Heaton-Johnson KJ, Master SR, Lee FS : Defective Tibetan PHD2 binding to p23 links high altitude adaption to altered oxygen sensing. J Biol Chem 289: 14656-14665, 2014

13) Xiang K, Ouzhuluobu, Peng Y, Yang Z, Zhang X, Cui C, Zhang H, Li M, Zhang Y, Bianba, Gonggalanzi, Basang, Ciwangsangbu, Wu T, Chen H, Shi H, Qi X, Su B : Identification of a Tibetan-specific mutation in the hypoxic gene EGLN1 and its contribution to high-altitude adaptation. Mol Biol Evol 30 : 1889-1898, 2013

14) Mishra A, Mohammad G, Thinlas T, Pasha MA: EGLN1 variants influence expression and SaO2 levels to associate with high-altitude pulmonary oedema and adaptation. Clin Sci (Lond) 124 : 479-489, 2013

15) Buroker NE, Ning XH, Zhou ZN, Li K, Cen WJ, Wu XF, Zhu WZ, Scott CR, Chen SH : EPAS1 and EGLN1 associations with high altitude sickness in Han and Tibetan Chinese at the Qinghai-Tibetan Plateau. Blood Cells Mol Dis 49 : 67-73, 2012

16) Hanaoka M, Droma Y, Basnyat B, Ito M, Kobayashi N, Katsuyama Y, Kubo K, Ota M : Genetic variants in EPAS1 contribute to adaptation to high-altitude hypoxia in Sherpas. PLoS ONE 7 : e50566. doi : 10.1371/journal. pone. 0050566, 2012

17) Zhao M, Kong QP, Wang HW, Peng MS, Xie XD, Wang WZ, Jiayang, Duan JG, Cai MC, Zhao SN, Cidanpingcuo, Tu YQ, Wu SF, Yao YG, Bandelt HJ, Zhang YP: Mitochondrial genome evidence reveals successful Late Paleolithic settlement on the Tibetan Plateau. Proc Natl Acad Sci U S A 106 : 21230-21235, 2009

18) Gilbert-Kawai ET, Milledge JS, Grocott MP, Martin DS : King of the mountains : Tibetan and Sherpa physiological adaptations for life at high altitude. Physiology (Bethesda) 29: 388-402, 2014

19) World Medical Association General Assembly: World Medical Association Declaration of Helsinki : ethical principles for medical research involving human subjects. J Int Bioethique 15 : 124-129, 2004

20) Kobayashi T, Koyama S, Kubo K, Fukushima M, Kusama S: Clinical features of patients with high-altitude pulmonary edema in Japan. Chest $92: 814-821,1987$

21) Savourey G, Guinet A, Besnard Y, Garcia N, Hanniquet AM, Bittel J : Evaluation of the Lake Louise acute 
mountain sickness scoring system in a hypobaric chamber. Aviat Space Environ Med 66 : 963-967, 1995

22) de Bakker PI, Yelensky R, Pe'er I, Gabriel SB, Daly MJ, Altshuler D : Efficiency and power in genetic association studies. Nat Genet 37 : 1217-1223, 2005

23) Tian H, McKnight SL, Russell DW : Endothelial PAS domain protein 1 (EPAS1), a transcription factor selectively expressed in endothelial cells. Genes Dev 11: 72-82, 1997

24) Guo SW, Thompson EA : Performing the exact test of Hardy-Weinberg proportion for multiple alleles. Biometrics $48: 361-372,1992$

25) Epstein AC, Gleadle JM, McNeill LA, Hewitson KS, O’Rourke J, Mole DR, Mukherji M, Metzen E, Wilson MI, Dhanda A, Tian YM, Masson N, Hamilton DL, Jaakkola P, Barstead R, Hodgkin J, Maxwell PH, Pugh CW, Schofield CJ, Ratcliffe PJ : C. elegans EGL-9 and mammalian homologs define a family of dioxygenases that regulate HIF by prolyl hydroxylation. Cell 107 : 43-54, 2001

26) 1000 Genomes Project Consortium, Abecasis GR, Altshuler D, Auton A, Brooks LD, Durbin RM, Gibbs RA, Hurles ME, McVean GA : A map of human genome variation from population-scale sequencing. Nature 467 : 1061-1073, 2010

27) Gorlov IP, Moore JH, Peng B, Jin JL, Gorlova OY, Amos CI : SNP characteristics predict replication success in association studies. Hum Genet 133 : 1477-1486, 2014

28) Phillips PC : Epistasis-the essential role of gene interactions in the structure and evolution of genetic systems. Nat Rev Genet 9 : 855-867, 2008

29) Droma Y, Hanaoka M, Ota M, Katsuyama Y, Koizumi T, Fujimoto K, Kobayashi T, Kubo K : Positive association of the endothelial nitric oxide synthase gene polymorphisms with high-altitude pulmonary edema. Circulation 106 : 826-830, 2002

30) Qi Y, Sun J, Zhu T, Wang W, Liu J, Zhou W, Qiu C, Zhao D : Association of angiotensin-converting enzyme gene insertion/deletion polymorphism with high-altitude pulmonary oedema: a meta-analysis. JRAAS 12: 617-623, 2011

31) Woods DR, Pollard AJ, Collier DJ, Jamshidi Y, Vassiliou V, Hawe E, Humphries SE, Montgomery HE : Insertion/ deletion polymorphism of the angiotensin I-converting enzyme gene and arterial oxygen saturation at high altitude. Am J Respir Crit Care Med 166 : 362-366, 2002

32) Luo Y, Zou Y, Gao Y: Gene polymorphisms and high-altitude pulmonary edema susceptibility : a 2011 update. Respiration $84:$ 155-162, 2012

33) Wan X, Yang C, Yang Q, Xue H, Fan X, Tang NL, Yu W : BOOST : A fast approach to detecting gene-gene interactions in genome-wide case-control studies. Am J Hum Genet 87 : 325-340, 2010

(2015. 1. 29 received; 2015. 2. 17 accepted) 